Patricia Whitelock and Russell Cannon, eds.

\title{
Proper Motions of Local Group Satellites
}

\author{
Mike Irwin \\ IoA, Madingley Road, Cambridge CB3 OHA, UK
}

\begin{abstract}
Many direct attempts to measure the proper motions of Galactic satellite galaxies are either in progress or have recently been completed. In this review I will summarise and examine the current state of knowledge in this field and will also highlight several indirect methods that can be used as alternative estimators or constraints on the proper motions of satellite galaxies throughout the Local Group. A good example of this latter approach is provided by the Sagittarius dwarf galaxy, where ongoing tidal disruption of the system has left a giant arc of debris tracing out its orbital path.
\end{abstract}

\section{Introduction}

Early attempts at directly measuring the proper motion of nearby spiral galaxies including M31, M51 and M101, by Curtis (1915), van Maanen (1916) and Lampland (1916), were doomed to failure for two fundamental reasons. First, even for Galactic satellite systems with expected typical transverse motions of $v_{t} \approx 200 \mathrm{~km} \mathrm{~s}^{-1}$ at canonical distances of $r \approx 100 \mathrm{kpc}$, the measurable proper motion $\mu$ is only

$$
\mu=0.42\left(\frac{v}{200 k m / s}\right)\left(\frac{r}{100 k p c}\right)^{-1} \operatorname{mas~yr}^{-1}
$$

corresponding to movement on a photographic plate of less than a few tenths of a micron every 10 years. Second, these pioneering efforts used faint Galactic stars for a reference frame and as pointed out by Eddington (1917) the signal from, and the uncertainties of, the reflex solar motion completely swamp the intrinsic motion of the galaxies. Precisely the same problems plague direct measurement of satellite proper motions now, although much recent progress has been made using measuring machines to digitise the extant plate material (see Cudworth 1997 for a comprehensive review).

Outlying members of the Local Group present even more difficulties with

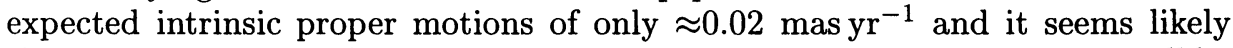
that space-based interferometric missions with micro-arcsecond precision will be needed to make progress here. Given these difficulties it is worthwhile exploring alternative approaches that we can use in either estimating or placing limits on the proper motion of satellite galaxies.

Let us start by looking at these alternatives. We will then be in a position to compare the "predictions" made by such methods with the best available measurements made from the direct approach. 


\section{Proper Motion \& Local Group Membership}

Traditionally, two criteria have been used to define Local Group membership for satellites beyond the Galactic retinue: (i) the distance from the barycentre of the Local Group - usually taken to be $\lesssim 1500 \mathrm{kpc}$ for membership; (ii) the measured radial velocity, corrected to a stationary centre-of-mass for the Local Group usually taken to be less than $50-100 \mathrm{~km} \mathrm{~s}^{-1}$ for membership. The latter measure is often displayed by plotting heliocentric velocity against the cosine of the angle between the object and the apex of the solar motion with respect to the Local Group (e.g. van den Bergh 1994). Although the radial velocities thus derived are not true Local Group-centric measures, the deprojection factor is generally between $(0.8-1.0)^{-1}$, small enough to be ignored given the uncertainties in the mass ratio of $\mathrm{M} 31: \mathrm{MW}$ and the distances.

Curiously, the two criteria are rarely combined directly. Figure 1 shows the Local Group plus noteworthy nearby galaxies in the $d_{g}-v-r_{g}$ plane. Satellites of our Galaxy and M31 are shown as small open squares and circles respectively and can be ignored since their kinematics are specific to the host galaxy rather than the Local Group as a whole. Superposed on the diagram are two pairs of symmetrically placed, nearly parallel, tracks which correspond to the Virial boundaries for purely radial motion and for total Local Group masses of $3.0 \times 10^{12}$ and $4.5 \times 10^{12} \mathrm{M}_{\odot}$ respectively. Alternatively, if the tangential velocity was similar to the radial velocity then the outer Virial boundary would be appropriate for a mass of $3.0 \times 10^{12} \mathrm{M}_{\odot}$. A mass ratio for $\mathrm{M} 31: \mathrm{MW}=2$ was used for this particular plot but lowering this to 1.5 makes no significant difference.

The additional "knotted" line introduces a third criterion derived from the timing argument which can also be used to constrain the transverse velocities. Originally applied by Kahn \& Woltjer (1959) to an assumed radial motion of M31 with respect to the Galaxy, this idea was extended by Lynden-Bell (1981) to include the motion of the outer satellites of the Local Group. Lynden-Bell showed that the extra information obtained by incorporating these additional data can be used to break the degeneracy between the total mass of the Local Group and its age. However, this works both ways. If the age of the Universe is, say, $13 \mathrm{Gyr}$, and the mass of the Local Group is $3.0 \times 10^{12} \mathrm{M}_{\odot}$, then the "knotted" line represents the maximum outer boundary that an object can have reached in that time given its currently observed radial velocity and distance. In particular, satellites such as IC 5152 and Sag DIG, must have undergone almost purely radial motion, while satellites lying to the left of the boundary such as, Aquarius ${ }^{1}$, Leo A, and WLM can be expected to have a modest transverse motion. Einasto \& Lynden-Bell (1982) showed how sensitive this "turnaround" time is to proper motion for the M31-MW interaction and by analogy, for the outer Local Group satellites. For example, an orbital eccentricity of 0.89 , corresponding to a low transverse velocity for M31 of $\approx 65 \mathrm{~km} \mathrm{~s}^{-1}$, adds 4 Gyr to the time taken to reach "turnaround". It is also noteworthy that with the "re-

\footnotetext{
${ }^{1}$ Aquarius has almost zero radial velocity with respect to the Local Group barycentre making it almost certainly a Local Group member. Previous estimates of its distance have ranged from $800 \mathrm{kpc}$ to $4 \mathrm{Mpc}$. I have used the latest value presented by Lee (1998) at this meeting.
} 
LOCAL GROUP ENVIRONS

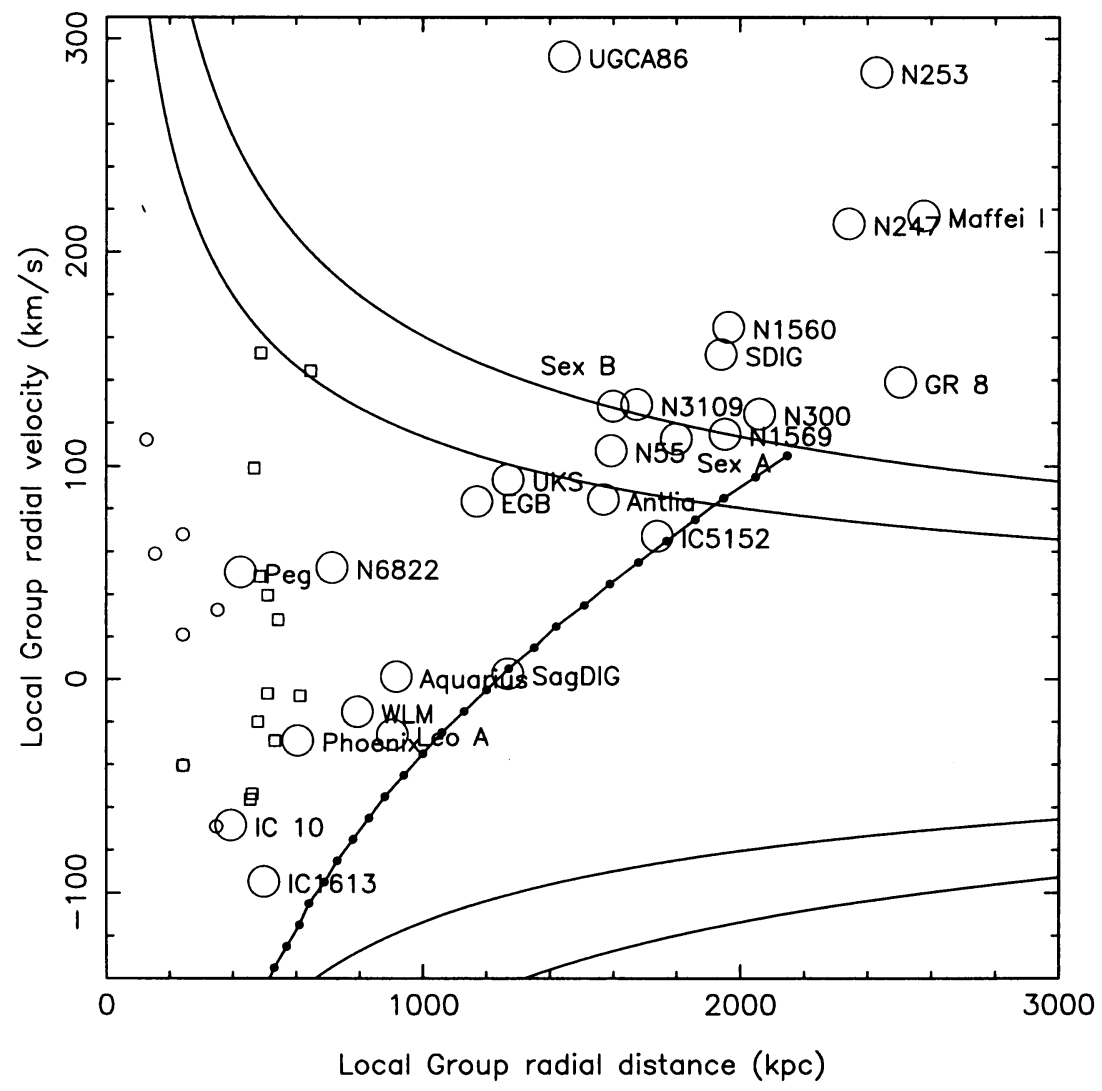

Figure 1. Local Group membership criteria: the upper and lower tracks are Virial boundaries for purely radial motion and Local Group masses of $3.0 \times 10^{12}$ and $4.5 \times 10^{12} \mathrm{M}_{\odot}$; the "knotted" line denotes the limiting distance attainable for an object with purely radial motion in a notional 13 Gyr since the Big Bang (see text for more details). The usual suspect Local Group members lie in the plausible Virial boundary zone and there is no straightforward boundary between the Local Group and prominent Sculptor Group members such as NGC 55 and NGC 300 - UKS 2323-326 could "belong" to either. The recently announced Local Group candidate Cam A (Gallart et al., these proceedings) lies within a few degrees on the sky of NGC 1560 and NGC 1569. Together with EGB $0427+63$ these galaxies probably constitute a small external grouping that merges further out with members of the M81 group. Sextans A, Sextans B, NGC 3109 and Antlia may also comprise another sub-group on the periphery. Although shown separately, IC 10, IC 1613 and Pegasus are most likely satellites of M31. Note that the isolated outer satellite, Tucana, is absent due to lack of a radial velocity, and both Antlia and Phoenix have only tentative $\mathrm{H}$ I velocities. 


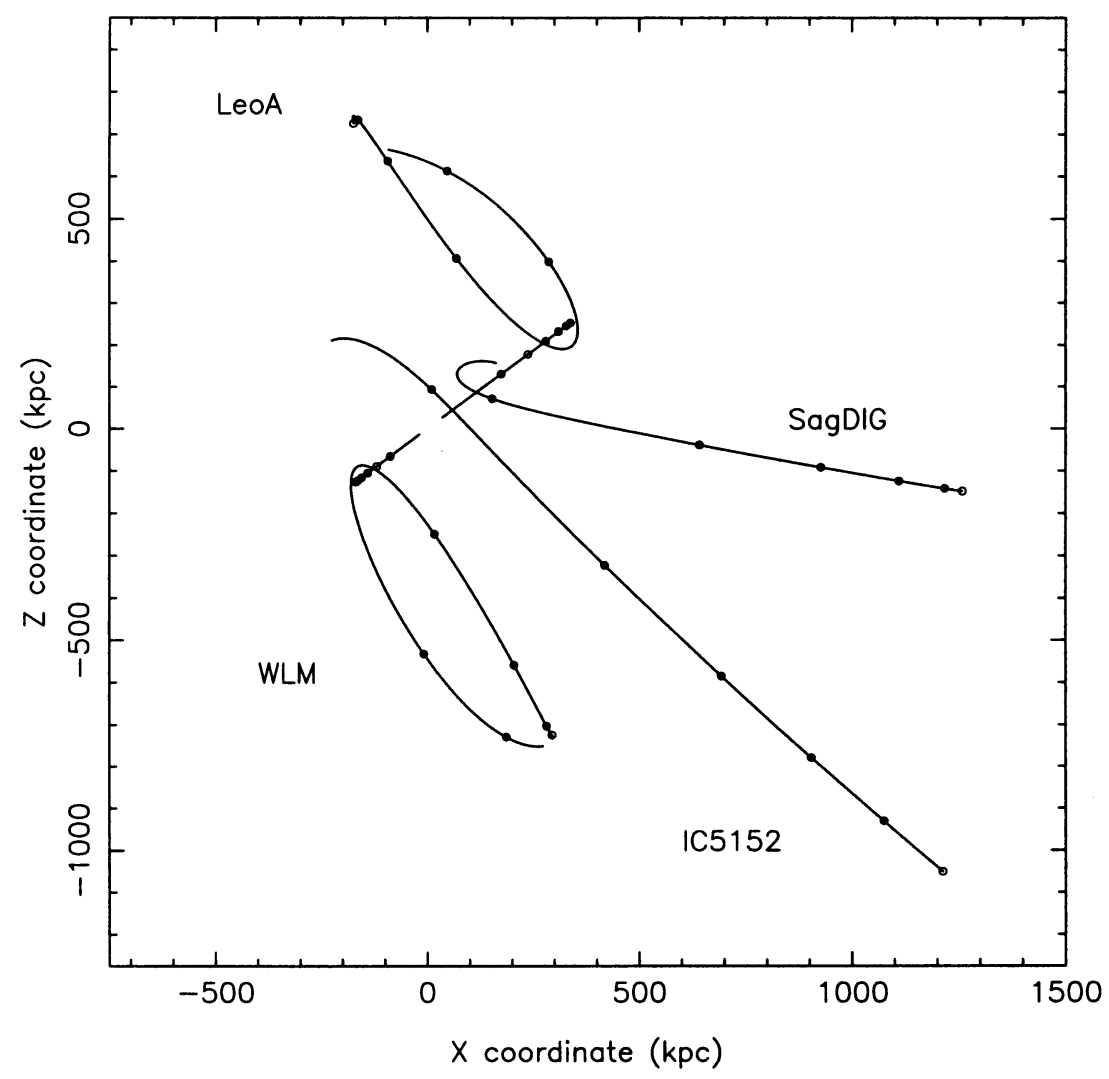

Figure 2. A projection of the reverse orbital tracks of a sample of outer Local Group satellites relative to the motion of M31-MW, derived assuming purely radial motion. The knots on the orbits are spaced every $2.5 \mathrm{Gyr}$, with the open knots representing the start.

moval" of Aquarius there are now no known nearby objects, bounded by the Virial surface and to the right of this boundary.

An example of assuming purely radial orbits for M31, MW, IC 5152, Sag DIG, WLM and Leo A, and using backward integration to track the evolution of the motion, is shown in Fig. 2. Note that as the smaller mass objects move inward radially, the time-varying equipotential surfaces of the two main mass concentrations induce non-radial motion of the satellites. For a Local Group mass of $4 \times 10^{12} \mathrm{M}_{\odot}, \mathrm{M} 31$, MW, IC 5152 and Sag DIG are within $100 \mathrm{kpc}$ of the centreof-mass 13 Gyr ago, while WLM and Leo A would require a modest transverse motion component to be close to this position. 


\section{Constraints on the Proper Motion of Galactic Satellites}

Historically, perhaps the first accurate attempt to infer the proper motion of a satellite galaxy from indirect measurements was made by Meatheringham et al. (1988), who used Feitzinger's method ${ }^{2}$ to estimate the transverse velocity of the LMC and obtained the value $V_{\mathrm{T}}=275 \pm 65 \mathrm{~km} \mathrm{~s}^{-1}$. The "ghostly streams" of Lynden-Bell \& Lynden-Bell (1995; and these proceedings) can be thought of as an extension of this approach to ordered motions around the Great Circle of the orbital track (see also section 3.2).

Tidal distortion, and eventually destruction, of satellite systems, provides direct information about satellite proper motions. It is this idea that permeates the method of "ghostly streams", but it is also more generally applicable. For example, various numerical studies (e.g. Oh, Lin \& Aarseth 1995) have shown that tidal disruption of a satellite galaxy proceeds by the formation of a leading tidal bridge and a trailing tidal tail that follow the original orbital path of the satellite. Initially, the tidal interaction introduces a progressive distortion in the projected appearance of a satellite along the direction of motion, such that the outer surface brightness contours are more elliptical than the central parts (Johnston et al. 1995a). When viewed from close to the Galactic centre we would therefore expect that the position angle of the major axis of a tidally distorted dwarf spheroidal (dSph) satellite closely defines the plane of the orbital track.

\subsection{The Plane of the Orbital Motion}

The surface brightness distribution of the Sculptor dwarf spheroidal (dSph) has exactly the characteristics expected from a tidally distorted system, with circular inner isophotes and progressively more elliptical outer isophotes (cf. Figs. 1 \& 3 of Irwin \& Hatzidimitriou 1995). The general surface morphology of the majority of the Galactic dSphs plus the presence of putative extra-tidal stars in most, if not all systems, strongly supports the view that Galactic tidal forces play an important role in determining their overall properties.

Sculptor is also a good candidate for a satellite system in the process of tidal disruption (see section 3.3) and as such we would expect its proper motion to lie along the direction defined by its major axis at PA $99^{\circ} \pm 1^{\circ}$. This apparently conflicts with the only measured proper motion available for Sculptor by Schweitzer et al. (1995) which has a PA $40^{\circ} \pm 24^{\circ}$. However, in this case the background reference frame used to define the proper motion consists of only six galaxies, three of which have large errors.

Another good example of a tidally distended system is provided by Ursa Minor. This dSph currently has the most accurately determined measured proper motion (Schweitzer et al. 1996) which aligns not only along the major axis direction, but is moving along an orbit trailing the Magellanic Clouds with similar specific orbital angular momentum and energy. This lends direct support to the idea of tidally-aligned streams of satellites systems first proposed by Lynden-Bell (1976).

\footnotetext{
${ }^{2}$ If a satellite covers a large angle on the sky and has predictable ordered internal motion, then the transverse velocity is reflected in the variation of radial velocity across the system. Unfortunately, the LMC is probably the only Galactic satellite to which this method is applicable.
} 


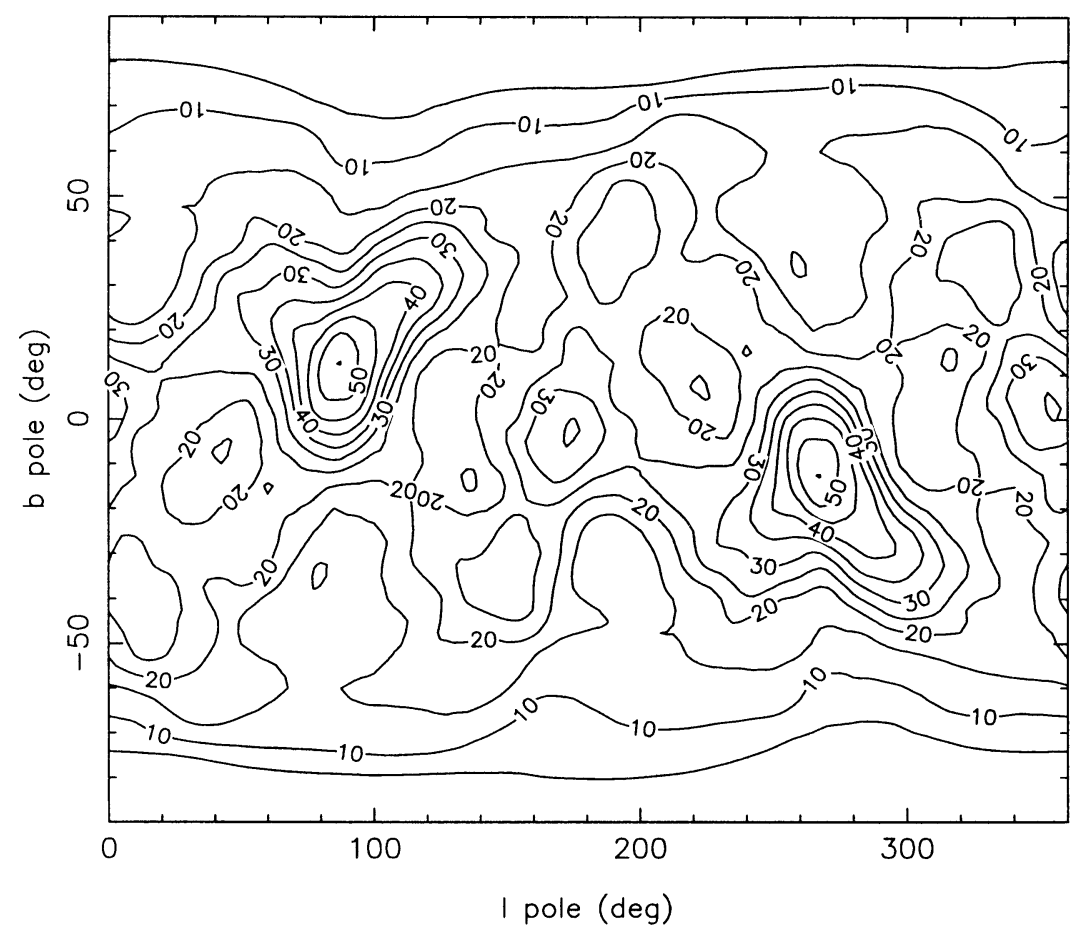

Figure 3. The contours represent the number of carbon stars from the APM survey, plus other previously known faint high latitude carbon stars, found within 10 degrees of putative Great Circle orbital paths. Each Great Circle track is defined by the Galactic coordinates of its pole. A 2.5 degree grid of these possible orbital poles was used as a basis for the contour map. The obvious concentration of Carbon stars at $l=90^{\circ}, b=14^{\circ}$ are coincident with the expected orbital pole of the Sagittarius dwarf galaxy. The observed $l, b$ of the carbon stars have been corrected to a Galactocentric coordinate system using approximate distances derived from their apparent $R$-band magnitudes.

\subsection{Great Circles of Debris}

After significant tidal disruption at a perigalactic passage, disintegration of a satellite is rapid and even over one orbit, leads to an enormous elongation of the dwarf along the orbital direction. Lateral spreading proceeds more slowly such that, in principle, it is still possible to trace the Great Circle path of the original orbit after several passages (Johnston et al. 1995b). A typical satellite galaxy has an orbital period $\approx 1 \mathrm{Gyr}$, therefore we might still expect to find coherent traces of dwarf galaxies captured several Gyr ago, although the typical lateral velocity dispersion of $\approx 10 \mathrm{~km} \mathrm{~s}^{-1}$ causes any stellar debris to spread over several degrees of sky in $\approx 1$ Gyr making it hard to find by direct searches.

Perhaps the most convincing example of the importance of such tidal effects comes from the recent discovery of the Sagittarius dSph (Sgr) by Ibata, Gilmore 
\& Irwin (1994). This system is probably in the final stages of tidal disruption and has not only contributed at least four "Halo" globular clusters to the Galaxy: M54; Ter 7; Ter 8; \& Arp 2; but also a giant arc of observable stellar debris. The discovery of cool carbon stars in the central region of the Sgr galaxy (Whitelock et al. 1996) highlights the potential for locating possible tidal debris of the Sagittarius dwarf using cool carbon stars as tracers.

As part of an ongoing systematic survey for faint high latitude carbon (FHLC) stars with $|b| \gtrsim 30^{\circ}$ and $11<R<17$, we have discovered over 50 cool AGB N-type carbon stars at distances ranging from 10 - $100 \mathrm{kpc}$ (Totten \& Irwin 1998). These intermediate-age carbon giants seem unlikely to have formed in the outer Galactic Halo in isolation from other star forming regions, and we have been investigating the possibility that they arose from the disruption of tidally captured dwarf satellite galaxies such as the Sagittarius dwarf. By using the method of Great Circle Counts (Johnston et al. 1995b) we find a significant excess of carbon stars associated with the Great Circle track of Sgr (see Fig. 3). We have accurate radial velocities from $1 \AA$ resolution optical spectroscopy and approximate distances to the carbon stars from $J H K$ photometry. This extra phase space information over a large fraction of the orbit constrains lot only the proper motion of Sgr but also provides a strong constraint on the orm of the Galactic potential.

In addition, the phase space constraints provided by the carbon stars can se used to assess the likelihood that other Halo globular clusters could have seen torn off Sgr and could therefore be expected to have closely related specific srbital angular momentum and energy (e.g. Lynden-Bell \& Lynden-Bell 1995) and hence predictable proper motions. Of the fifty or so Halo clusters with $r_{G C}>$ $10 \mathrm{kpc}$, the four mentioned previously lie within the main body of Sgr whilst three others are within 10 degrees of the orbital track, and have distances and radial velocities consistent with membership of Sgr: Pal 12 - a well established "young" Halo cluster with properties resembling Ter 7 , lies only $30^{\circ}$ downstream of the main body of Sgr; Pal 2 - an old Halo cluster on the opposite side of the Galaxy to Sgr; NGC 2419 - the most distant $\left(r_{G C}=92 \mathrm{kpc}\right)$ of the luminous Galactic clusters.

\subsection{Kinematic Constraints on Orbital Parameters}

It is a moot point whether any of the Galactic dSphs are in tidal equilibrium, however, the majority of the stellar radial distributions are well fitted by single component King (1962) models with tidal truncation. Given the mass potential of the Galaxy, the observed tidal radius then provides a direct estimate of the perigalactic distance and hence the orbital ellipticity and proper motion. Likewise, the observed radial velocity is clearly a lower bound to the maximum radial velocity of the orbit and as pointed out by Oh, Lin \& Aarseth (1995) this constraint defines a minimum orbital eccentricity and hence maximal proper motion.

A logarithmic Halo potential of the form $\phi=v_{c}^{2} \ln \left(r / r_{c}\right)$, is a reasonable approximation for all the Galactic dSphs bar Sagittarius. If the observed radial velocity in such a potential, $v_{r}$, at Galactic distance, $r$, is the maximum radial velocity attained, then it is straightforward to show that the specific angular momentum, $l$, is given by $l_{e_{m i n}}=v_{c} r$, the equivalent proper motion by 
Table 1. Kinematic Properties of Galactic dSphs

\begin{tabular}{|c|c|c|c|c|c|c|c|c|c|}
\hline Galaxy & ${ }^{\mathrm{R}}$ & c) ${ }^{r_{t}}$ & $\begin{array}{c}\mathrm{V}_{r} \\
\left(\mathrm{~km} \mathrm{~s}^{-1}\right)\end{array}$ & $\mathrm{e}_{t}$ & $\mathrm{e}_{\min }$ & $\mathrm{R}_{p}$ & $\mathrm{R}_{a}$ & $\begin{array}{l}\mu_{t} \\
(\mathrm{~m} z\end{array}$ & $\begin{array}{l}\mu_{e_{m i n}} \\
\left.\mathrm{yr}^{-1}\right)\end{array}$ \\
\hline Carina & 89 & 0.58 & 7 & 0.59 & 0.02 & $\begin{array}{l}23 \\
87\end{array}$ & $\begin{array}{l}89 \\
91\end{array}$ & 0.23 & 0.52 \\
\hline Draco & 76 & 0.50 & -94 & 0.70 & 0.30 & $\begin{array}{l}15 \\
58\end{array}$ & $\begin{array}{c}84 \\
106\end{array}$ & 0.22 & 0.61 \\
\hline Fornax & 133 & 2.08 & -36 & 0.39 & 0.12 & $\begin{array}{c}59 \\
119\end{array}$ & $\begin{array}{l}136 \\
150\end{array}$ & 0.22 & 0.35 \\
\hline Leo I & 230 & 0.65 & 177 & 0.83: & 0.53: & $\begin{array}{l}29: \\
143\end{array}$ & $\begin{array}{c}324: \\
463\end{array}$ & 0.06 & 0.20 : \\
\hline Leo II & 219 & 0.49 & 22 & $0.85:$ & 0.07 & $\begin{array}{l}18: \\
205\end{array}$ & $\begin{array}{l}221: \\
237\end{array}$ & 0.04 & 0.21 \\
\hline Sculptor & 78 & 1.33 & 74 & - & 0.23 & $\overline{63}$ & - & - & 0.60 \\
\hline Sextans & 91 & 3.10 & 73 & - & 0.23 & $\overline{73}$ & $\overline{117}$ & - & 0.51 \\
\hline Ursa Minor & 66 & 0.63 & -88 & 0.53 & 0.28 & $\begin{array}{l}23 \\
51 \\
\end{array}$ & $\begin{array}{l}74 \\
90 \\
\end{array}$ & 0.39 & 0.70 \\
\hline
\end{tabular}

$\mu_{e_{\min }}=v_{c} / 4.74 r$ mas $\mathrm{yr}^{-1}$ and that this also defines the minimum orbital eccentricity, $e_{\min }$. This can be a surprisingly tight constraint as shown by the derived estimates for the orbital parameters of the Galactic dSphs in Table 1. The estimates of the perigalactic and apogalactic distances in the table correspond to the tidal (upper entry) and minimum eccentricity (lower entry) orbits. Note particularly that the final column gives a tight upper limit for the expected proper motion for the assumed form of the Galactic potential. For example, compare the upper bound of $0.70 \mathrm{mas} \mathrm{yr}^{-1}$ for the proper motion of Ursa Minor with the recently measured value of $0.64 \pm 0.13$ (see Table 2). Interestingly, both Sculptor and Sextans have tidal radii extending well beyond any long term stable configuration, hence the gaps in the table for $e_{t}$ and the corresponding orbital extrema, $R_{p}, R_{a}$. Both of these dSphs are therefore excellent candidates for systems that are actively being tidally disrupted.

\section{Direct Measurement of Proper Motion}

Modern direct methods generally rely on defining an external reference frame based on distant background galaxies. It is usually the systematic errors in defining this reference frame with respect to the programme stars, that dominates the proper motion errors (e.g. Schweitzer et al. 1995), rather than the random errors from measuring the positions of the individual stars. Another source of systematic error comes from contaminating foreground stars which can contribute a significant extra component caused by the reflex solar motion.

Table 2 summarises the various direct measures, uncorrected for reflex solar motion, of satellite galaxies made so far. The majority were undertaken using photographic plates and measuring machines. Photographic plates have the advantage of large baselines, in practice up to 40 years, but suffer from serious residual systematic astrometric errors (i.e. after all the reduction) at the $\approx 10$ mas level for single pairs of 1 st and 2 nd epoch Schmidt plates, and at the $\approx 5$ mas level 
Table 2. Directly Measured Proper Motions of Galactic Satellites

\begin{tabular}{|c|c|c|c|c|c|c|c|}
\hline Galaxy & $\mu_{\alpha}$ & $\left.\mathrm{r}^{-1}\right)^{\mu_{\delta}}$ & $\mathrm{V}_{\mathrm{T}}(\mathrm{km}$ & $-1)^{V_{R}}$ & Method & $\begin{array}{l}\Delta T \\
(\mathrm{yr})\end{array}$ & Ref. \\
\hline Draco & $0.6 \pm 0.4$ & $1.1 \pm 0.5$ & & & 4 Schmidt plates & 27 & 1. \\
\hline Sculptor & $0.73 \pm 0.22$ & $-0.07 \pm 0.25$ & $198 \pm 100$ & $95 \pm 40$ & 26 Assorted plates & 40 & 2. \\
\hline Sagittarius & $\begin{array}{c}-2.8 \pm 0.8 \\
-2.65 \pm 0.08\end{array}$ & $\begin{array}{c}-1.4: \\
-0.88 \pm 0.08\end{array}$ & $278 \pm 20$ & $137 \pm 5$ & $\begin{array}{l}6 \text { Schmidt plates } \\
\text { HST WFPC2 }\end{array}$ & $\begin{array}{c}35 \\
2\end{array}$ & $\begin{array}{l}3 . \\
4 .\end{array}$ \\
\hline Ursa Minor & $\begin{array}{c}0.5 \pm 0.8 \\
0.06 \pm 0.08\end{array}$ & $\begin{array}{c}1.2 \pm 0.5 \\
0.07 \pm 0.10\end{array}$ & $188 \pm 20$ & $-91 \pm 20$ & $\begin{array}{l}4 \text { Schmidt plates } \\
39 \text { KPNO } 4 \mathrm{~m} \text { plates }\end{array}$ & $\begin{array}{l}27 \\
40\end{array}$ & $\begin{array}{l}1 . \\
5 .\end{array}$ \\
\hline LMC & $\begin{array}{c}1.20 \pm 0.28 \\
1.3 \pm 0.6 \\
1.94 \pm 0.29\end{array}$ & $\begin{array}{c}0.26 \pm 0.26 \\
1.1 \pm 0.7 \\
-0.14 \pm 0.36\end{array}$ & $215 \pm 48$ & $48 \pm 41$ & $\begin{array}{l}21 \text { CTIO 4m plates } \\
35 \text { PPM stars } \\
36 \text { Hipparcos } \\
\text { stars }\end{array}$ & 14 & $\begin{array}{l}6 . \\
7 . \\
8 .\end{array}$ \\
\hline $\mathrm{SMC}$ & $\begin{array}{l}0.92 \pm 0.20 \\
1.23 \pm 0.84\end{array}$ & $\begin{array}{l}-0.69 \pm 0.20 \\
-1.21 \pm 0.75\end{array}$ & $155 \pm 55$ & $46 \pm 30$ & $\begin{array}{l}20 \text { AAT } 4 \text { m plates } \\
11 \text { Hipparcos } \\
\text { stars }\end{array}$ & 20 & $\begin{array}{l}9 . \\
8 .\end{array}$ \\
\hline
\end{tabular}

1. Scholz \& Irwin (1994); 2. Schweitzer et al. (1995); 3. Irwin et al. (1996a); 4. Ibata et al. (1998); 5. Schweitzer et al. (1996); 6. Jones et al. (1994); 7. Kroupa, Röser \& Bastion (1994); 8. Kroupa \& Bastion (1997); 9. Irwin et al. (1996b)

for plates from $4 \mathrm{~m}$ telescopes. Clearly, combining whole series of plates helps to reduce these errors, but in practice a proper motion error of $\approx 0.1 \operatorname{mas~yr}^{-1}$ represents the limit of this approach (e.g. Schweitzer \& Cudworth 1996). As noted by Kroupa \& Bastion (1997), even this level of accuracy is not adequate to decide, for example, if the SMC is bound to the LMC.

CCDs, because of their larger dynamic range (1000:1) compared to photographic plates (30:1), enable more accurate measurement of individual object positions (10mas -v-100mas). The drawbacks here are the generally much shorter baseline available, coupled with the difficulty of retaining the same instrumental setup - to minimise subtle systematic errors - for a long enough period.

A possible way forward here is to use HST+WFPC2, where individual object astrometric errors of $\approx 2$ mas are achievable. In particular the baseline and stability offered by WFPC2 using the HST archive for 1st epoch data, coupled with new observations over the next few years, could enable accurate, $10 \%$, proper motions to be obtained for a large number of Galactic Halo clusters and satellite galaxies. An example of the potential offered by HST in this field is the proper motion determination for Sgr by Ibata et al. (1998) listed in Table 2, using a two year baseline of HST data.

Accurate proper motions for just a few of these Halo systems would greatly enhance our understanding of the Halo, leading to better constraints on formation models, and subsequent evolution of the Halo. 


\section{References}

Cudworth, K. 1997, in: Proper Motions and Galactic Astronomy, (ed.) R.M. Humphreys,ASP Conf. Ser. 127, p. 91

Curtis, H. 1915, PASP, 27, 214

Eddington, A. 1917, MNRAS, 77, 375

Einasto, J., Lynden-Bell, D. 1982, MNRAS, 199, 67

Ibata, R., Gilmore, G., Irwin, M.J. 1994, Nature, 370, 194

Ibata, R., Irwin, M.J., Lewis, G. 1998, in preparation

Irwin, M.J., Hatzidimitriou, D. 1995, MNRAS, 277, 1354

Irwin, M.J., Ibata, R., Gilmore, G., Wyse, R., Suntzeff, N. 1996a, in: The Formation of the Galactic Halo...Inside and Out, (eds.) H. Morrison \& A. Sarajedini, ASP Conf. Ser. 92, p. 841

Irwin, M.J., Demers, S.D., Kunkel, W.E., Malin, D. 1996b, AAS, 188.6510

Johnston, K.V., Spergel, D.N., Hernquist, L. 1995a, ApJ, 451, 598

Johnston, K.V., Bolte, M., Hernquist, L. 1995b, in: Formation of the Galactic Halo...Inside and Out, (eds.) H. Morrison \& A. Sarajedini, ASP Conf. Ser. 92 , p. 483

Jones, B.F., Klemola, A.R., Lin, D.C.N. 1994, AJ, 107, 1333

Kahn, F.D., Woltjer, L. 1959, ApJ, 130, 705

King, I.R. 1962, AJ, 67, 471

Kroupa, P., Röser, S., Bastion, U. 1994, MNRAS, 266, 412

Kroupa, P., Bastion, S. 1997, New Astro., 2, 77

Lampland, C. 1916, Pop. Astron., 24, 667

Lynden-Bell, D. 1976, MNRAS, 174, 695

Lynden-Bell, D. 1981, Observatory, 101, 111

Lynden-Bell, D., Lynden-Bell, R.M. 1995, MNRAS, 275, 429

Meatheringham, S.J., Dopita, M.A., Ford, H.C., Webster, B.L. 1988, ApJ, 327, 651

Oh, K.S., Lin, D.N.C., Aarseth, S.J. 1995, ApJ, 442, 142

Schweitzer, A.E., Cudworth, K.M., Majewski, S.R., Suntzeff, N.B. 1995, AJ, 110,2747

Schweitzer, A.E., Cudworth, K.M. 1996, AAS, 188.0901

Scholz, R.D., Irwin, M.J. 1994, in: Astronomy From Wide Field Imaging, (eds.)

H.T. MacGillivray \& E.B. Thompson, IAU Symp. 161, p. 535

Totten, E., Irwin, M.J. 1998, MNRAS, 294, 1

van den Bergh, S. 1994, AJ, 107, 1328

van Maanen, A. 1916, ApJ, 44, 210

Whitelock, P.A., Irwin, M., Catchpole, R.M. 1996, New Astro., 1, 57 


\section{Discussion}

Zaritsky: What is your estimate of the initial luminosity/mass of Sagittarius?

Irwin: Approximately $50 \%$ of the mass of Sgr appears to be in a great circle stream away from the main body. This gives a total luminosity for Sgr of $\mathrm{M}_{V} \approx-15 \mathrm{mag}$.

Terndrup: Do the carbon stars lie in the regions of the sky which should have the highest expected surface density from the models?

Irwin: The expected number density as a function of azimuthal angle from the models varies qualitatively just as the distribution of $\mathrm{C}$ stars. With the caveat that in the SGC the survey is still not complete.

Whitelock: In agreement with what you have just said Mike - several of the C-stars do seem to be Miras. Mira lifetimes are only about $2 \times 10^{5} \mathrm{yr}$; therefore there must be a very large number of main sequence stars to go with them.

Aparicio: For UMi, we apparently find no stars at a distance of $\simeq 2^{\circ}$ from the center of the galaxy (that corresponds to about $2 \mathrm{kpc}$ at the distance of UMi). Do you think this is reasonable?

Irwin: The tidal disruption models predict an orbital track spreading of stars which is a function of the mass ratio of the satellite to the mass of the disrupting body interior to the orbit. For example, for a mass ratio of $10^{-3}$ a spread of $10 \%$ per orbit could occur. For Ursa Minor we expect at most a spread of $\approx \pm 1^{\circ}$ per orbit if it has been significantly tidally distorted. So your result is not too surprising.

Lloyd Evans: Can you tell us about the spectra of these carbon stars?

Irwin: The carbon stars are apparently normal ' $\mathrm{N}$ '-type from $J H K$ photometry and optical spectroscopy results. Cool AGB stars are preferentially selected by the $B_{J}-R \geq 2.5$ colour criterion. 